\title{
OBESITY, INFLAMMATION, AND T-CELL METABOLISM
}

\author{
K. Hristov* \\ Department of Chemistry and Biochemistry, Medical Faculty, Medical University, Sofia, \\ Bulgaria
}

\begin{abstract}
Aim: The overview of the interdependence of the immune system and the system metabolism. Regulation of metabolism is immunomodulatory, and targeting key cellular metabolic enzymes impacts T-cell development, altering the immune functions.

Background: The diet, gastrointestinal microbiota and the balanced function of liver, adipose and muscle tissues underlie the immune ecology. Chronic inflammation (macrophage, TH1, and TH17 T-cell infiltration) associates with obesity, and the development of metabolic syndrome, cardiovascular diseases, type 2 diabetes, IBD and intestinal malignancies. While naive T-cells use beta-oxidation, TCA cycle, and mitochondrial respiration to produce ATP, activated T-cells, similarly to cancer cells, employ the Warburg's effect to power their function. The development of T-cells depends on key metabolic regulators, like mTORC1 (TH1 and TH17 T-cells) and mTORC2 (TH2 T-cells). Inhibition of HIF1-alpha (critical for TH17 T-cells) results in the development of FOXP3+ Treg T-cells, improving autoimmune disorders. Metabolic flexibility of normal cells underlines the successful treatment of neoplastic, autoimmune and hyper-sensitivity disorders.

Conclusions: The immune system influences the system metabolism, and depends on the function of adipose tissue, muscles, liver, pancreas, lungs and gastro-intestinal tract. Diet and pharmacological regulation of T-cell metabolic activity influence immune function during autoimmunity, infections, and vaccinations.
\end{abstract}

Key Words: T-cells, lymphocyte signaling, glycolysis, glutaminolysis, apoptosis, metabolism, inflammation.

\begin{abstract}
ABBREVIATIONS
AMPK, AMP-activated protein kinase; ConA, concanavalin A; DFMO, difluoro-methylornitine; DHA, decosahexenoic acid; eEF2alpha, eukaryotic elongation factor 2 alpha; EPA, eicosapentaenoic acid; HIF1-alpha, hypoxia-inducible factor 1 alpha; IBD, inflammatory bowel disease; iNOS, inducible nitric oxide synthase; LC-n-3-PUFA, longchain n-3 poli-unsaturated fatty acids; NADPH, nicotine-amid adenine di-nucleotide phosphate (reduced); NO, nitric oxide; PGE2, prostaglandin E2; PPP, pentose phosphate pathway; ROS, reactive oxygen species; SLC1A1, Na2+-dependent glutamine symporter; SLC1a5, glutamine importer; SLC7A11, glutamine-cysteine antiporter.
\end{abstract}

\footnotetext{
*Correspondence to: Kroum K. Hristov, Department of Chemistry and Biochemistry, Medical Faculty, Medical University - Sofia, Sofia - 1431, Bulgaria; E-mail:4747868@dir.bg
}

\section{AIM}

The aim of this review article is to overview the interactions and interdependence of the immune system and

the system metabolism. Diet and specific pharmacological inhibitors of liver, muscles, adipose tissue metabolism are immunomodulatory regulators. Specific metabolic profiles characterise naive, activated, and memory T-cells, and mTOR, MYC and HIF1-alpha activities are essential for metabolic reprogramming. Restriction of nutrients inhibits T-cell and immune function. Increased muscle protein degradation during injury, infection, burns, and surgical interventions is necessary for the maintenance of the immune system. Skeletal muscles, not diet, supply lymphocyte metabolism with glutamine. Further, key T-cell metabolic enzymes and signaling regulators are promising therapeutic targets for many autoimmune disease models, including 
autoimmune diabetes, IBD, asthma, and EAE, during infections, and vaccinations.

\section{DIET AND INFLAMMATION}

Immune system, scanning the body, depends and influences the function of adipose tissue, muscles, liver, pancreas, lungs and gastrointestinal tract. Chronic inflammation parallels obesity, metabolic syndrome, cardiovascular disease, type 2 diabetes, IBD, and colon cancer (1). While visceral adipose tissue produces pro-inflammatory factors (free fatty acids and adipokines), triggering the release of liver cytokines and coagulation factors (ultimately resulting in liver insulin resistance, type 2 diabetes, and non-alcoholic fatty liver), the inflammatory response during IBD (ulcerative colitis and Crohn disease) is associated with hypertrophy of visceral adipose tissue around the inflamed intestines (2-5). Obese mice have increased TH1/ TH2 T-cell ratio. Obese people increase both pro-inflammatory $\mathrm{TH} 1$ and cytotoxic T-cells, and anti-inflammatory $\mathrm{TH} 2$ and Treg T-cells in subcutaneous adipose tissue. Visceral adipose tissue in obese humans has more anti-inflammatory T-cells (3). Adipose tissue macrophages switch to proinflammatory phenotype (5). Weight loss decreases macrophage infiltration of white adipose tissue, and improves inflammatory cytokine profiles. A number of systemic biologically active regulators influence the inflammatory response during obesity, altering insulin resistance (TNF-alpha, IL6), migration and adhesion of monocytes and macrophages, hunger, and energy expenditure. IL6 regulates TH17/TH22 T-cell in adipose tissue, decreases the glucose uptake in skeletal muscles, and the hepatocyte responsiveness to insulin. CCL5 and IL7, regulating T-cell survival and proliferation, increase from emaciated to nonobese to obese people $(1,3,6)$.

Adiponectin has anti-inflammatory function: decreases hepatocyte gluconeogenesis (through phosphoenolpyruvate carboxykinase and glucose-6-phosphatase), and up-regulates fatty acid catabolism in the skeletal muscles. Adiponectin is anti-atherogenic, downregulating TNF-alpha and endothelial adhesion molecules (1, 7). Statins (thiazolidinedions) have anti-inflammatory effect, improving glucose tolerance. They decrease resistins levels in obese animals (8). Statins decrease adipocyte TNF-alpha production and induce adiponectin. They regulate endothelial function and leukocyte migration. The anti-diabetic (Pravastatin) and anti-inflammatory effects depend on NF-kappa-B activity inhibition in the liver and adipose tissue (1). The specific expression of IKK-beta (NF-kappa-B signaling regulator) in liver and adipose tissue, but not in the skeletal muscles highlights the insulin resistance. IL17 and IL22 secreting CD4+ Tcells in the adipose tissue inhibit insulin signaling and hepatocyte metabolism. Insulin fails to trigger AKT-S473 phosphorylation and activation, but activates JNK. This increases hepatocyte glucose production. IL17 and IL22 prevent the inhibitory effect of insulin on gluconeogenesis and aerobic glycolysis. JNK stabilises IRS1-S307 phosphorylation. JNK $\mathrm{KO}$ mice are not resistant to insulin when obese. JNK inhibition improves hepatocyte insulin sensitivity in type 2 diabetes model. JNK inhibitors and IL17 neutralisation improve liver steatosis and inflammation, decreasing TH17 T-cells infiltration (3). Aspirin inhibits not only IKK and JNK signaling, but also other $\mathrm{S} / \mathrm{T}$ kinases, participating in TNF-alpha-induced insulin resistance. Aspirin acts as an anti-oxidant, decreasing NF-kappa-B and AP1 activation. Salicylates decrease insulin resistance of obese animal models, and high doses salicylates improve insulin sensitivity in type 2 diabetes patients.

While LC-n-6-PUFA (long-chain poliunsaturated fatty acids) in the diet are proinflammatory, LC-n-3-PUFA beneficially influence insulin secretion, and decrease lipid levels. LC-n-3-PUFA have both metabolic and immunomodulatory effects. LC-n-6-PUFA (arachidonic acid) to LC- n-3-PUFA (EPA, DHA) ratio in the human diet varies between 17:1 to 1:1. Mice on high-fat/LC-n-3-PUFA diet have less adipose tissue, small adipocytes, improved insulin levels, lower levels of liver unsaturated fatty acids and triacylglycerols, altered liver and brown fat gene expression, immune cell activation, decrease of intestinal endothelial cell activity, increase of pro- (TNFalpha, MCP1) and anti-inflammatory (IL10) cytokines, increase of M1(CD11c)/M2(CD206) macrophage ratio in visceral adipose tissue, decrease of $\mathrm{CD} 4 / \mathrm{CD} 8$-alpha ratio in visceral adipose tissue and spleen. High-fat/LC-n-3PUFA diet decrease liver lipogenesis and increases liver peroxisomal beta-oxidation; increases UCP1, and T- and B-cell activation in visceral adipose tissue; decreases the expression of NOS2 and CDKN1A; stimulates T-cell differentiation (JAGGED2 and E3 ubiquitin ligase WWP1); increases IL7-regulated B-cell differentiation; increases pro-inflammatory SPP1 and IFN-gamma, regulating TH1 T-cell differentiation. LC-n-3-PUFA potentiates the 
immunosupressive effect of T-cells. Highfat/LC-n-3-PUFA diet increases the expression of ICAM1, VCAM1 and TEK in small intestinal blood and lymph vessels, regulating endothelial cell activation, adhesion, and remodeling of extracellular matrix. Further LC-n-3-PUFA increase spleen weight, due to the increased erythrocyte turnover (5).

\section{METABOLIC CHANGES DURING T- CELL'S DIFFERENTIATION}

Specific bioenergetic and biosynthetic activities characterise naive, activated, and memory Tcells. ERK and AKT signaling pathways funnelling extracellular stimuli to critical transcriptional regulators, like mTOR, MYC and HIF1-alpha, essential for metabolic reprogramming $(9,10)$.

Naive, quiescent T-cells employ aerobic mitochondrial phosphorylation of glucose, amino acids and lipids for the production of ATP. IL7R signaling and autophagy maintain naive $\mathrm{T}$-cell survival. T-cell activation increases the expression of glucose and amino acid transporters, and transferrin receptors. Restriction of nutrients down-regulates T-cell function, and immune system in general (10). The build-up of cellular biomass during the initial growth and proliferation following stimulation with ConA, phytohaemaglutinin and anti-CD3/anti-CD28 antibodies depends on switch to anabolic metabolism. T-cells reprogram their metabolism from beta-oxidation of fatty acids and pyruvate oxidation in the TCA cycle to aerobic glycolysis, glutaminolysis and pentose-phosphate pathway (PPP), constituting the Warburg's effect. While glutamine (trough the TCA cycle) and glucose (through PPP) consumption increases, carnitine decreases - reflecting the decreased mitochondrial beta-oxidation. Metabolites associated with glycolysis, glutaminolysis, and biosynthetic pathways (pyruvate, lactate, citrate and aspartate) are abundant. This is in contrast with those used in TCA cycle (alphaketoglutarate, succinate, fumarate), which are present in trace amounts in proliferating cells, reflecting the decreased metabolic flow $(\sim 50 \%)$ through pyruvate dehydrogenase and the TCA cycle. Glucose maintains the cellular survival. Glutamine is essential for T-cell growth and proliferation, and its scarcity is overcome with the addition of nucleotides and polyamines (11). T-cell stimulation does not alter the function of isocitrate dehydrogenase 3 and malic enzyme (12).

Glycolysis is the main metabolic pathway for energy production in activated T-cells (Figure 1) similarly to cancer cells, proliferating T-cells depend on Warburg's effect (anaerobic glycolysis to lactate) for ATP production, and metabolites for PPP (glucose-6-phosphate) and phospholipid synthesis (glycerol-3-phosphate). T-cells have a constant, low levels of expression of hexokinase 1 and phosphofructokinase phosphatase, and high expression levels of glyceraldehyde-3-phosphate dehydrogenase, phosphoglycerate kinase 1 , enolase $1 / 2$, pyruvate kinase $\mathrm{M} 2$, and lactate dehydrogenase $\mathrm{A} / \mathrm{B}$.

The low activity of TCA cycle coincides with increased expression of PDK1/3/4, and inhibited activity of pyruvate dehydrogenase and oxoglutharate dehydrogenase. The positive regulators of TCA cycle: aconitase 2, succinate tiokinase/succinyl-CoA synthase 2, succinate dehydrogenase 2 and isocitrate dehydrogenase 2 are expressed in significant amounts. They participate in the reductive carboxylation of alpha-ketoglutarate. These genes are regulated by K-RAS, and participate in the metabolic reprogramming (12).

PPP uses glucose-6-phosphate (glucose-6phosphate dehydrogenase and 6phosphogluconate dehydrogenase) to supply reduced NADPH for lipid and nucleotide synthesis, and anti-oxidative stress reactions. Anabolic metabolism increases the concentration of ribose-5-phosphate and erythrose-4-phosphate, used for nucleic and amino acid synthesis. If not used in the synthetic pathways, these metabolites are utilised in glycolysis for energy production (13).

Glutaminolysis secures the biosynthetic reactions in peripheral T-cells. Although significant in quiescent lymphocytes, glutaminolysis increases furthermore following activation. Glutaminolysis is a directional pathway, saturated with substrate. It parallels the increase of glutamine concentration in the blood. Glutamine, generated in the intestinal lumen is utilised by the enterocytes. Most of the glutamine in the blood is generated in the skeletal muscles. Thus, T-cells glutamine metabolism depends on skeletal muscles. Increased muscle protein degradation during injury, infection, burns, and surgical interventions are necessitates the activity of the immune system, and the cellular recovery mechanism (14).

Glutamine is metabolised through glutamine transaminase to glutamate (Figure 1), not through glutamate dehydrogenase 1 (14). Lymphocyte glutamine synthetase/glutaminase 1 contributes $30 \%$ of the ammonia originating from glutamine. Unlike ileal enterocytes, producing alanine, lymphocytes generate aspartate from glutamine (aspartate aminotransferase - 70\%; alanine aminotransferase - $3 \%$ ). 


\section{HRISTOV $K$.}

Phosphoenolpyruvate carboxykinase generates phosphoenolpyruvate from oxaloacetate during glutamine oxidation. This includes the activity of malat dehydrogenase and oxaloacetate decarboxylase that use malate for the generation of oxaloacetate and pyruvate. Most of the pyruvate, generated from glutamine, is used for the production of lactate. The partial oxidation of glutamine to lactate is quantitatively the most important pathway for glutamine utilisation in lymphocytes. The complete oxidation of glutamine to $\mathrm{CO} 2$ is achieved through 2- oxoglutarate in a sequence of 5 decarboxylating reactions (twice through oxoglutharate dehydrogenase and once through isocitrate dehydrogenase 3 in the TCA cycle; and once through phosphoenolpyruvate carboxykinase and pyruvate dehydrogenase). Generation of acetylCoA from pyruvate depends on the amount of the later, and the activity of pyruvate dehydrogenase. The low levels of acetyl-CoA produced from pyruvate are not demonstrative for low activity of the TCA cycle.

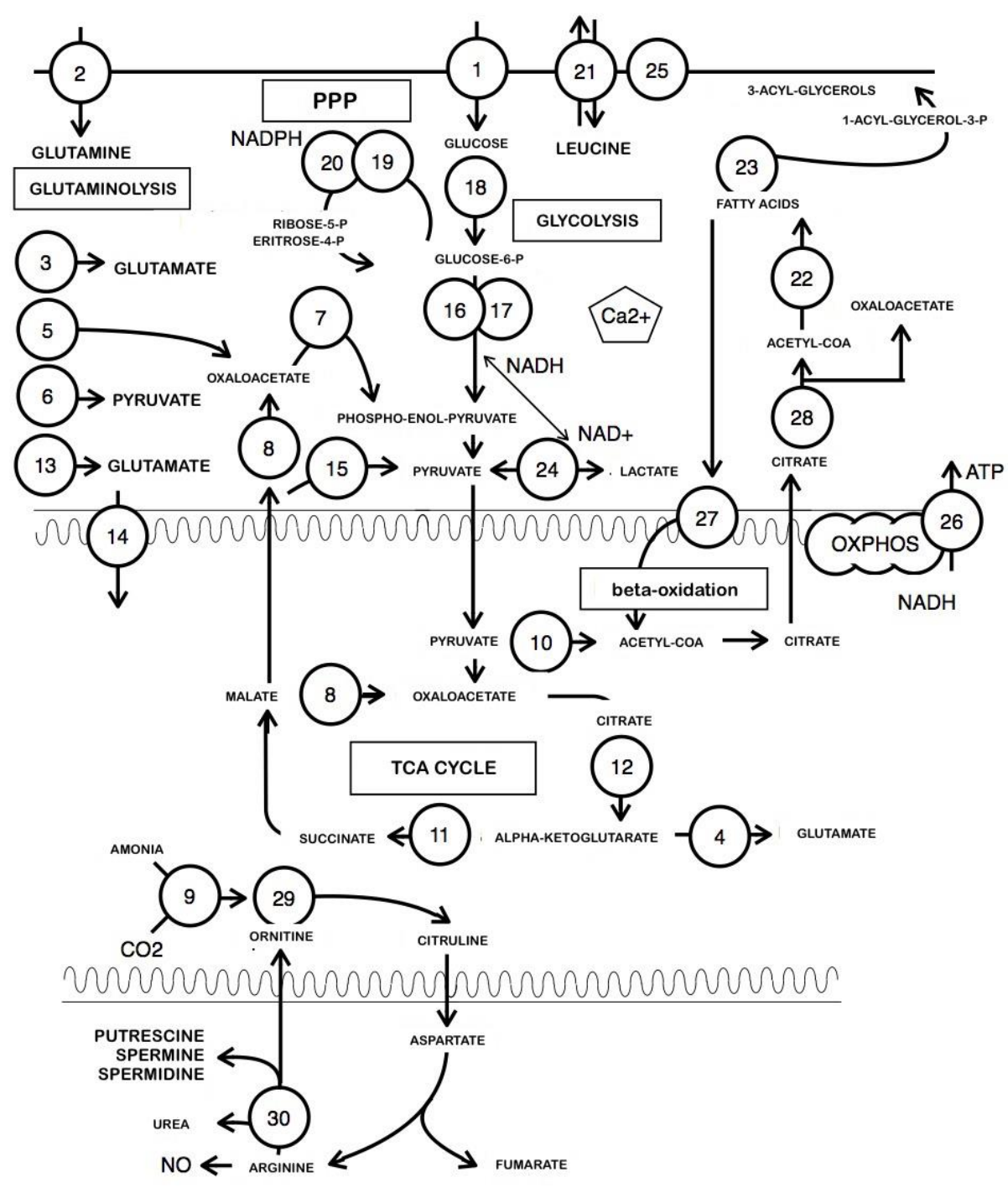

Figure 1. The main enzymes involved in the T-cell metabolism during differentiation and proliferation: 1 . GLUT1, glucose transporter 1; 2. SLC1A5, glutamine transporter; 3. glutamine transaminase; 4. glutamate dehydrogenase; 5. aspartate aminotransferase; 6. alanine aminotransferase; 7. phosphoenolpyruvate carboxykinase, oxaloacetate decarboxylase; 8. NADP+-malate dehydrogenase; 9. carbomoylphosphate synthetase; 10. pyruvate dehydrogenase; 11 . alpha-ketoglutarate dehydrogenase; 12 . isocitrate dehydrogenase; 13. glutaminase; 14. glutamine mitochondrial transporter; 15. malic enzyme; 16. fructose-2,6-bisphosphatase; 17. 6-phosphofructo-1-kinase; 18. hexokinase 1; 19. glucose-6-phosphate dehydrogenase; 20. 6phosphogluconate dehydrogenase; 21. CD98, SLC7A5-SLC3A2, glutamine-leucin antiporter, transporter of neutral AA; 22. acethyl-CoA carboxylase; 23. glycerol-phopshate acyl-transferase; 24. lactate dehydrogenase A; 25. CD71, transferin receptor; 26. ATP synthase; 27. carnitine-palmitoil transferase-I; 28, ATP-citrate lyase; 29. ornithine transcarbomoylase; 30 . arginase. 
Lymphocyte activation increases glutamine metabolism, which results in intracellular depletion of glutamine and 2-oxoglutarate. The enzymes (plasma membrane and mitochondrial glutamine transporters, glutaminase, oxoglutharate dehydrogenase and phosphoenolpyruvate carboxykinase) comprising the glutamine metabolism are regulated. Activatory signalling increases the number of glutamine transporters, and changes their oxidative state. ConA increases cytosolic and mitochondrial calcium, activating oxoglutharate dehydrogenase and glutamate dehydrogenase 1, and subsequently glutaminase/glutamine synthetase 1 activity. The decrease of 2oxoglutarate stimulates glutamine transport through mitochondrial membrane. Phosphoenolpyruvate carboxykinase and oxoglutharate dehydrogenase activities increase simultaneously, securing big fluctuations in malate and oxoglutarate concentrations. Immune stimulation results in decrease of serum glutamine concentration.

The need of glutamine and branched-chain amino acids increases following injury, surgery, infection and burns. One of the results of protein catabolism is to supply nitrogen and carbon for the glutamine synthesis. However, most of the muscle glutamine is not utilised by lymphocytes. Glutamine metabolises 4 times faster in comparison with the glycolysis, supplying 10 $37 \%$ of the fuel for respiration. Although glutamine synthetase 1 has an increased activity in injured rats, the flow through the oxidative part of glutamine metabolism is higher in comparison to the biosynthetic part, in both resting and proliferating lymphocytes. Glutamine oxidation is only partial, similarly to glucose oxidation to lactate. Despite the energy loss, glycolysis and glutaminolysis comprise a simple and effective control mechanism, swiftly securing macromolecular synthesis when the cell needs it.

MYC activity regulates glutaminolysis, increasing the expression of glutamine importer SLC32A1, SLC32A2, and the enzymes, comprising the glutaminolytic pathway. Decreased expression of SLC1A5 in transformed cells results in insignificant reduction of glutamine import, suggesting a role for alphaketoglutarate in aspartate production through the oxidative metabolism. The increased expression of glutaminase 2 in proliferating murine T-cells is independent of p53, while human cell lines depend on p53 for glutaminase 2 induction (11). SLC1A1 is a sodium-dependent simporter of glutamine and branched-chain amino acids, like leucine, which together with glutamine antiporter
SLC7A5-SLC3A2 induces mTORC1 activity. Intracellular glutamine itself does not induce mTORC1 activity. Leucine activates RAGRHEB-mTOR pathway. Without glutamine, the leucine flow decreases, and mTOR activity is inhibited.

The lack of glutamine inhibits proliferation only of T-cells, which is partially overcome following the addition of dimethyl-aspartate and dimethyl-alpha-ketoglutarate. Aminotransferase activity, metabolising glutamine to aspartate, supports the malignant growth. Aminotransferase inhibition with amino-oxy-acetate prevents proliferation. This inhibition is overcome with dimethyl-aspartate (but not with dimethyl-alpha-ketoglutarate). EGCG-induced cell death is blocked with dimethyl-alpha-ketoglutarate. Further, glutamine supplies the cells with anti-oxidants, like glutathione, protecting against the proapoptotic effects of accumulating ROS. Thus, supporting anabolic and survival roles for glutamine in T-cells (12).

Fatty acids are an important fuel even for animals in a fed state. They supply beta-oxidation (Figure 1) and TCA cycle, providing more than $50 \%$ of the energy in lymphocytes. BetaOxidation is important for naive quiescent $\mathrm{T}$ cells, and is down-regulated following activation. Etomoxir, an inhibitor of carnitine-palmitoyl transferase1a, does not influence T-cell proliferation. Contrary to this, inhibition of glucose and glutamine catabolism (hexokinase 1 with 2DG, glucose-6-phosphate dehydrogenase with dehydroepiandrosterone, glutaminase 1 with DON, and transaminase inhibition with aminooxy-acetate) prevents T-cell proliferation. mTOR inhibition with rapamycin decreases glycolysis, equally increasing beta-oxidation. mTOR inhibition prevents the synthesis of fatty acids and complex lipids decreasing the expression of acetyl-CoA carboxylase and/or mitochondrial glycerol-phosphate acyl-transferase (11). While effectors T-cells have glycolytic phenotype during acute inflammation, T-cells during chronic inflammation, like SLE, depend on the mitochondrial oxidative phosphorylation. The increased activity of mTORC1 stabilises and triggers the generation of new mitochondria. mTOR inhibition decreases, independently of S6K, mitochondrial function. mTORC1 regulates mitochondrial gene expression through YY1 and PGC1a transcriptional regulators. Starvation or mTOR inhibition decreases the number of mitochondria, and induces autophagy. 
Autophagy is a catabolic process for energy production and survival, where starved and stressed cells metabolise their own organelles and cytoplasm. Autophagy is associated with the accumulation of LC3B-coated vesicles. mTORC1 activity inhibits this process, phosphorylating ULK1:ATG13:FIP200 complex. The lack of ULK1 makes cells resistant to rapamycin-induced autophagy (13). ATG7 is critical for mitochondrial degradation in the thymus. LC3B-coated vesicles accumulation accompanies the increased glycolysis during T-cell activation. The lack of ATG7 and BECLIN1 increase cellular survival following serum withdrawal. The lack of ATG5 results in T-cell apoptosis following their stimulation in the periphery. Inhibition of autophagy results in inability of T-cells to proliferate due to disregulation of ER integrity and calcium homeostasis.

\section{T-CELL DIFFERENTIATION}

The thymus provides environment for the sequential development of T-cells - from DN, $\mathrm{DP}$, to CD4+ and CD8+ SP T-cells. NOTCH through mTORC2, NF-kappa-B and FOXO, and pre-TCR controls early thymocyte development and transition of DN into DP T-cells. RICTOR is essential for cellular growth and glycolysis in thymocytes. Although mTOR is critical for APC and B-cell differentiation and function, RICTOR is specifically essential only for thymus development, and B-cell and myeloid cell development is unaffected (10). Activated, peripheral T-cells depend on aerobic glycolysis, glutaminolysis and leucine metabolism, comprising the effect of Warburg (Table 1). TCR stimulation without co-stimulatory signalling results in tolerance - anergy and induced cell death. Simultaneous CD28 costimulation triggers the production of IL2 and proliferation. Activated T-cells differentiate to TH1, TH2, TH17 and Treg T-cells (13)

Table 1. T-cell metabolic regulators: mTOR, PTEN, LKB1, calcium, p53, HIF1, MYC, and ERR-alpha.

\begin{tabular}{|c|c|}
\hline & T-CELL METABOLIC REgulATORS: \\
\hline mTOR & $\begin{array}{l}\text { an essential environmental sensor, critical for the development of autoimmunity and malignant transformation. mTORC1 consists of RAPTOR, mLST8/GbL, PRAS40 and DEPTOR. mTORC1 activity is } \\
\text { inhibited by TSC1/ TSC2 through RHEB. TCS2 is regulated by PI3K-AKT and AMPK. mTORC1 regulates S6K1 and 4E-BP1, and ultimately - cellular growth and proliferation. Rapamycin inhibits } \\
\text { mTORC1 only. mTORC2 consists of RICTOR, mSIN1, PROTOR1, mLST8 and DEPTOR. mTORC2 is regulated by AKT, GSK1 and PKC-alpha, following its complexing with ribosomes. TCR and } \\
\text { CD28, ICOS and OX40 co-stimulation positively regulates PI3K-mTOR pathway. CTLA4 and PD1 are negative regulators of PI3K-AKT-mTOR pathway. AMPK activity following cellular stress, and } \\
\text { REDD1 triggered by hypoxia inhibit mTOR. The lack of mTOR does not affect quiescent T-cells. mTOR inhibition induces T-cell anergy }(10,13,15) \text {. }\end{array}$ \\
\hline PTEN & $\begin{array}{l}\text { a negative regulator of PI3K. The lack of PTEN allows T-cells to overcome the need of IL7R and pre-TCR stimulation. PTEN deficient thymocytes have an increased expression of MYC, AKT and } \\
\text { mTOR activities, which results in peripheral T-cell spontaneous activation and development of lymphomas and autoimmunity (10). }\end{array}$ \\
\hline LKB1 & $\begin{array}{l}\text { a ST kinase, active in hereditary malignant Peutz-Jeghers syndrome. LKB1-AMPK regulate metabolism through mTOR. While the lack of LKB1 impairs thymocyte development DN3 stage, AMPK1- } \\
\text { alpha deficiency results only in minor alterations of T-cell homeostasis (10). Specific inhibition of LXR, another T-cell regulator, results in increase of TCR-mediated cholesterol synthesis, and clonal } \\
\text { expansion. }\end{array}$ \\
\hline Calcium & $\begin{array}{l}\text { regulates metabolic reprogramming activating calcineurin, NFAT, PKC, CAMK and cytoskeletal proteins. T-cell activation regulates CRAC channels influencing mitochondrial metabolism, and ROS } \\
\text { production. Mitochondrial ROS and calcium regulate DRAK2 protein kinase, mediating T-cell activation, tolerance and homeostasis (16). }\end{array}$ \\
\hline p53 & $\begin{array}{l}\text { links metabolism and cellular survival. Glycolysis (GLUT1 and HK1) regulates anti-apoptotic signalling through AMPK, PKC-delta and p53, increasing the expression of PUMA and BIM. Glycolysis } \\
\text { inhibits acetylation and activation of p53 (17). Glycolysis inhibition increases the sensitivity to kinase inhibitors. Imatinib, a tyrosine kinase inhibitor, decreases glycolysis, and induces p53-dependent } \\
\text { apoptosis in BCR-ABL transformed cell lines. }\end{array}$ \\
\hline HIF1 & $\begin{array}{l}\text { along with MYC, PPAR-alpha, PPAR-gamma and SREBP, is regulated by MTOR. Hypoxia results in HIF1-alpha accumulation and transcriptional activity, increasing the expression of the glycolytic } \\
\text { enzymes. HIF1-alpha deficiency in T-cells fails to influence glycolysis and T-cell activation (10). REDD1 inhibits mTOR and HIF1-alpha (17). AT38, a NO donor, irreversibly inhibits cellular respiration, } \\
\text { proliferation, and apoptosis. The accumulation of lactate augments glycolysis. The up-regulated expression of glucose transporter } 1 \text { and phosphofructokinase } 1 \text { supports the energy-demanding } \\
\text { apoptotic mechanisms (18). }\end{array}$ \\
\hline MYC & $\begin{array}{l}\text { a repressor of TSC2, and activator of mTOR (11). mTORC1-4EBP- elF4e, and AKT-mTOR-AIP4-ITCH-mediated degradation of JUNB regulates MYC. MYC is essential for the maximal increase of } \\
\text { glycolysis (glucose transporter 1, hexokinase 2, pyruvate kinase M2, transketolase and glucose-6-phosphate dehydrogenase) following T-cell activation. MYC regulates glutamine fructose-6-phosphate } \\
\text { transaminase 1, carbamoyl-phosphate synthase, phosphoribosyl-pyrophosphate amidotransferase and ornithine aminotransferase, participating in glutaminolysis, synthesis of hexosamine, pyrimidines, } \\
\text { purines, and polyamines. While MYC regulates the expression of glutamine synthetase } 1 \text { - essential for glutaminolysis, p53 regulates glutamine synthetase } 2 \text { expression. While mTOR and AMPK are } \\
\text { not affected by the lack of MYC, end-effectors (like p70S6K, 4E-BP and S6) activity is blocked. CD98 (SLC3a2/SLC7a5) expression is impaired in MYC-deficient T-cells (11). DFMO (ornithine } \\
\text { decarboxylase and polyamine synthesis inhibitor) inhibits proliferation of activated T-cells, although the urea cycle enzymes, including arginase, are not induced in activated T-cells. Aldehyde } \\
\text { dehydrogenase 18A1, proline dehydrogenase, ornithine aminotransferase and ornithine decarboxylase, spermine synthase, and spermidine synthase metabolising glutamine and proline into ornithine } \\
\text { and polyamines, are induced by MYC in activated T-cells (11). }\end{array}$ \\
\hline ERR-alpha & induced after TCR co-stimulation, and regulates mTOR and AMPK, effector T-cell differentiation and development of autoimmune diseases (10). \\
\hline
\end{tabular}

TH1 CD4+ T-cells express IFN-gamma and TBET, and their development depends on IL12 and STAT4. They are highly glycolytic and the addition of fatty acids suppresses IFN-gamma production. The lack of RHEB inhibits mTORC1 activity and prevents TH1 and TH17 T-cell differentiation, decreasing autoimmune neuroinflammation. The lack of PPAR-alpha 
increases the production of IFN-gamma, increasing the pathology of EAE in male animals. The induced PPAR-gamma activity decreases IL2 and IFN-gamma secretion, suggesting a promising therapeutic target in many autoimmune disease models, including autoimmune diabetes, IBD, asthma, and EAE. These T-cells are not only anti-inflammatory, but fail to induce autoimmune disease following transfer in lymphopenic hosts $(10,13)$.

TH2 CD4+ T-cells express GATA3, IL4, IL5 and IL13, important for the immune responses against parasites. TH2 T-cells depend on mTORC2, and PPAR-gamma and RICTOR activation prevents cytokine production. TH2 Tcells have significantly higher levels of autophagy, compared to TH1 T-cells. mTORC1 inhibits autophagy. The ability of CD4+ T-cells to differentiate into $\mathrm{TH} 2 \mathrm{~T}$-cells depends on the lack of RHEB (mediating inhibitory signalling to mTORC1), and their ability to regulate autophagy $(10,13)$.

TH17 CD4+ T-cells contribute for the protection against bacterial infections, and the induction of tissue inflammation, and autoimmunity. TGFbeta, IL6, mTORC1 and ROR-gamma-t are essential for TH17 T-cell function. HIF1-alpha is selectively expressed by TH17 T-cells, and its knock-out or inhibition with 2DG supports the generation of iTreg T-cells. This prevents the autoimmune neuroinflammation in mice. HIF1alpha signalling contributes for the degradation of FOXP3. LXR/SREBP-dependent lipid metabolism, fatty acids and PPAR-gamma inhibit the development of TH17 T-cells $(10,13)$.

Treg T-cells differentiate following suboptimal TCR stimulation, or co-stimulation with TGFbeta (iTreg, induced Treg T-cells) or IL35 (Treg35 T-cells). Treg T-cells have oxidative metabolic profile - they metabolise fatty acids and utilise the mitochondrial respiration for energy production. TCR stimulation during the absence mTOR activity (with 2DG or rapamycin inhibition) results in the generation of FOXP3+ Treg T-cells. beta-Oxidation inhibition with etomoxir (an inhibitor of CPT1a) prevents the development of Treg T-cells. PPAR-alpha and PPAR-gamma, in the presence of TGF-beta and fatty acids in the medium, contribute for the differentiation of CD4+ CD25- T-cells into FOXP3+ Treg T-cells. HIF1 activity prevents the development of FOXP3+ Treg T-cells. Treg Tcells have low activity of mTOR, and mTOR inhibition contributes for the development of Treg T-cells, and increased responsiveness to TGF-beta. PD-L1 and S1PR1 prevent iTreg Tcell differentiation. Inhibition of amino acid metabolism and the absence of essential amino acids in the medium inhibit mTORC1 activity, inducing anergy and FOXP3 expression $(10,13)$.

Memory CD8+ T-cells: mTOR inhibition with rapamycin (AICAR, 2DG or NALA), and the inhibited expression of RAPTOR results in anergy, and supports the generation of memory CD8+ T-cells. Rapamycin treatment during active lymphocytic choriomeningitis virus infection increases antigen-specific memory CD8+ T-cells, without affecting the ability of the infected animals to clear the pathogen. These cells survive transfer in animal recipients. Rapamycin treatment inhibits TBET, and stabilises EOMES, eomesodermin, increasing tumour immunity. Homeostatic proliferating memory CD8+ T-cells, expressing MHCIautopeptide, IL7 and IL15, demonstrate significant anti-tumour potential. They depend on mTOR signalling. TRAF6 through AMPK, or AMPK activation with metformin control betaoxidation, and the differentiation of memory CD8+ T-cells. Memory T-cells phenotype is associated with an increase number of mitochondria and oxygen consumption. IL15 regulates oxidative metabolism, increasing mitochondrial biogenesis, and the expression of CPT1a. Rapamycin treatment supports T-cell migration to the secondary lymphoid tissues, but not to the sites of inflammation. This is accompanied with increased expression of CCR7, CD62L and S1PR1 in antigen-stimulated CD8+ T-cells (10, 13). Pharmacological regulation of mTOR activity provides effective therapeutic practicabilities to influence the function of memory T-cells, induced during vaccinations or infections.

\section{CONCLUSIONS}

The immune system has a dynamic and interactive relationship with adipose tissue, muscles, liver, pancreas, lungs and gastrointestinal tract. Alimentary constituents (like LCn-3-PUFA), and specific pharmacological inhibitors of JNK, NF-kappa-B and AP1 (like aspirin, thiazolidinediones, statins, and IL17 neutralisation) are metabolic (anti-adipogenic and insulin-sensitising) and immunomodulatory (anti-inflammatory) regulators.

Specific metabolic profiles characterise naive, activated, and memory T-cells. The activities of mTOR, MYC and HIF1-alpha are essential for metabolic reprogramming. While naive, quiescent T-cells employ aerobic mitochondrial phosphorylation for the production of ATP, activated T-cells, similarly to cancer cells, depend on Warburg's effect (aerobic glycolysis, glutaminolysis and PPP) for energy production. beta-Oxidation, important for quiescent T-cells, is down-regulated following activation. Glycolysis and autophagy maintain T-cell 
survival. Restriction of nutrients inhibits T-cell and immune function. Skeletal muscles, not diet, supply lymphocyte metabolism with glutamine. Increased muscle protein degradation during injury, infection, burns, and surgical interventions is necessary for the maintenance of the immune system.

Metabolic enzyme expression and activity are differentially regulated during T-cell differentiation. Key T-cell metabolic enzymes and signaling regulators are promising therapeutic targets for many autoimmune disease models, including autoimmune diabetes, IBD, asthma, and EAE. Inhibition of hexokinase 1 (with 2DG), glucose-6-phosphate dehydrogenase (with dehydroepiandrosterone), glutamine synthetase 1/glutaminase (with DON), and transaminase (with amino-oxy-acetate) blocks activation-induced metabolic reprogramming, and prevents T-cell proliferation. Glycolysis inhibition (with Imatinib, a tyrosine kinase inhibitor) augments the effect of other cellular signaling inhibitors. LXR/SREBP-dependent lipid metabolism, fatty acids and PPAR-gamma inhibit the development of pro-inflammatory TH17 T-cells. Inhibition of autophagy prevents T-cells proliferation. Inhibition of HIF1-alpha expression (with 2DG) in TH17 T-cells supports the generation of iTreg T-cells. Similarly, inhibition mTORC1 activity (with rapamycin, AICAR, 2DG or NALA) induces T-cell anergy and FOXP3 expression. Pharmacological regulation of $\mathrm{T}$-cell metabolic activity provides an effective therapeutic practicabilities influencing immune function during autoimmunity, infections, and vaccinations.

\section{ACKNOWLEDGEMENTS}

The author would like to thank the staff of Central Medical Library, Sofia, Bulgaria for the help during the preparation of this manuscript.

\section{REFERENCES}

1. Bastard JP, Maachi M, Lagathu C, Kim MJ, Caron M, Vidal H, Capeau J, Feve B. Recent advances in the relationship between obesity, inflammation, and insulin resistance. Eur Cytokine Netw, 17(1):4-12, 2006.

2. Fabbrini E, Higgins PB, Magkos F, Bastarrachea RA, Voruganti VS, Comuzzie AG, Shade RE, Gastaldelli A, Horton JD, Omodei D, Patterson BW, Klein S. Metabolic response to high- carbohydrate and lowcarbohydrate meals in a nonhuman primate model. Am J Physiol Endocrinol Metab, 304(4):E444-51, 2013.

3. Fabbrini E, Cella M, McCartney SA, Fuchs A, Abumrad NA, Pietka TA, Chen Z, Finck BN, Han DH, Magkos F, Conte C, Bradley D, Fraterrigo G, Eagon JC, Patterson BW,
Colonna M, Klein S. Association between specific adipose tissue CD4+ T-cell populations and insulin resistance in obese individuals. Gastroenterology, 145(2):36674.e1-3, 2013.

4. Kojima M, Tachibana N, Yamahira T, Seino S, Izumisawa A, Sagi N, Arishima T, Kohno M, Takamatsu K, Hirotsuka M, Ikeda I. Structured triacylglycerol containing behenic and oleic acids suppresses triacylglycerol absorption and prevents obesity in rats. Lipids Health Dis, 9:77, 2010.

5. Ludwig $\mathrm{T}$, Worsch $\mathrm{S}$, Heikenwalder $\mathrm{M}$, Daniel H, Hauner H, Bader BL. Metabolic and immunomodulatory effects of $n-3$ fatty acids are different in mesenteric and epididymal adipose tissue of diet-induced obese mice. Am J Physiol Endocrinol Metab, 304(11):E1140-56, 2013.

6. Leighton B, Budohoski L, Lozeman FJ, Challiss RA, Newsholme EA. The effect of prostaglandins E1, E2 and F2 alpha and indomethacin on the sensitivity of glycolysis and glycogen synthesis to insulin in stripped soleus muscles of the rat. Biochem $J$, 227(1):337-40, 1985.

7. Ludwig MS, Minguetti-Câmara VC, Heck TG, Scomazzon SP, Nunes PR, Bazotte RB, Homem de Bittencourt PI Jr. Short-term but not long-term hypoglycaemia enhances plasma levels and hepatic expression of HSP72 in insulin-treated rats: an effect associated with increased IL-6 levels but not with IL-10 or TNF- $\alpha$. Mol Cell Biochem, 397(1-2):97-107, 2014.

8. Rodríguez-Perea AL, Montoya CJ, Olek S, Chougnet CA, Velilla PA. Statins increase the frequency of circulating $\mathrm{CD} 4+\mathrm{FOXP} 3+$ regulatory $\mathrm{T}$ cells in healthy individuals. $J$ Immunol Res, :762506, 2015.

9. Ardawi MS, Newsholme EA. Metabolism in lymphocytes and its importance in the immune response. Essays Biochem, 21:1-44, 1985.

10. Yang K, Chi H. mTOR and metabolic pathways in $\mathrm{T}$ cell quiescence and functional activation. Semin Immunol, 24(6):421-8, 2012.

11. Wang R, Dillon CP, Shi LZ, Milasta S, Carter $\mathrm{R}$, Finkelstein D, McCormick LL, Fitzgerald $\mathrm{P}$, Chi H, Munger J, Green DR. The transcription factor Myc controls metabolic reprogramming upon $\mathrm{T}$ lymphocyte activation. Immunity, 35(6):871-82, 2011.

12. Gaglio D, Metallo CM, Gameiro PA, Hiller K, Danna LS, Balestrieri C, Alberghina L, Stephanopoulos G, Chiaradonna F. Oncogenic K-Ras decouples glucose and glutamine metabolism to support cancer cell growth. Mol Syst Biol, 7:523, 2011. 
13. Waickman AT, Powell JD. mTOR, metabolism, and the regulation of $\mathrm{T}$-cell differentiation and function. Immunol Rev, 249(1):43-58, 2012.

14. Newsholme EA, Crabtree B, Ardawi MS. Glutamine metabolism in lymphocytes: its biochemical, physiological and clinical importance. Q J Exp Physiol, 70(4):473-89, 1985.

15. Waickman AT, Powell JD. Mammalian target of rapamycin integrates diverse inputs to guide the outcome of antigen recognition in $\mathrm{T}$ cells. J Immunol, 188(10):4721-9, 2012.

16. Fracchia KM, Pai CY, Walsh CM. Modulation of $\mathrm{T}$ Cell Metabolism and
Function through Calcium Signaling. Front Immunol, 4:324, 2013.

17. Mason EF, Zhao Y, Goraksha-Hicks P, Coloff JL, Gannon H, Jones SN, Rathmell JC. Aerobic glycolysis suppresses p53 activity to provide selective protection from apoptosis upon loss of growth signals or inhibition of BCR-Abl. Cancer Res, 70(20):8066-76, 2010.

18. Hammami I, Bertrand M, Chen J, Bronte V, De Crescenzo G, Jolicoeur M. Nitric oxide affects immune cells bioenergetics: long-term effects of nitric-oxide derivatives on leukaemic Jurkat cell metabolism. Immunobiology, 217(8):808-15, 2012. 